\title{
BOSA: Binary Orientation Search Algorithm
}

\author{
Mohammad Dehghani, Zeinab Montazeri, Om Parkash Malik, Gaurav Dhiman, Vijay Kumar
}

\begin{abstract}
Optimization algorithms have many applications in solve the optimization problems in various sciences. The Orientation Search Algorithm (OSA) is an optimizer that is simulated the rules of a game called orientation game. In OSA searcher agent are players of orientation game that moves on the playground depending on the direction of the referee. in this study, binary model of OSA which called Binary Orientation Search Algorithm (BOSA) is proposed. BOSA and eight other algorithms (BGA, BPSO, BGSA, BGOA, BBA, BMOA, and $B D A)$ are tested on twenty-three Benchmark test function. Proposed BOSA has a high ability to solve optimization problems compared to other algorithms according to the evaluation results.
\end{abstract}

Keywords : Binary, Binary Orientation Search Algorithm Orientation Search, optimization.

\section{INTRODUCTION}

Each optimization problem consists of three parts: primary goals, secondary goals and decision variable. purpose of optimization is to discover the most suitable answer among possible solutions, somehow that first, observe the constraints of the problem and then optimize the problem [1]. Evolutionary algorithms have high ability to solve optimization problems. In this regard many optimization algorithms has been applied by the scholars to solve optimization problems in various science such as energy $[2,3]$, power engineering $[4,5]$, and energy carriers $[6,7]$.

Physics-based algorithms are kind of optimization algorithms that simulates the laws of physics. Simulated Annealing (SA) [8] is one of this class which mimics the process of gradual heating and cooling of metals. Spring Search Algorithm (SSA) [9, 10] is modeled on Hooke's law. Gravitation Search Algorithm (GSA) simulated the law of gravity. Some of other physics-based algorithms are: Ray Optimization (RO) algorithm, Magnetic Optimization Algorithm (MOA), and Black Hole (BH).

Evolutionary-based algorithms are another kind of optimization algorithms that simulates the birth process.

Revised Manuscript Received on November 08, 2019.

* Mohammad Dehghani

Mohammad Dehghani*, Dept. of Electrical and Electronics Engineering, Shiraz University of Technology, Shiraz, Iran. Email: adanbax@gmail.com

Zeinab Montazeri, Dept. of Electrical and Electronics Engineering, Shiraz University of Technology, Shiraz, Iran. Email: z.montazeri.2002@gmail.com

Om Parkash Malik, Department of Electrical Engineering, University of Calgary, Calgary Alberta Canada. Email: maliko@ucalgary.ca

Gaurav Dhiman, Department of Computer Science, Government Bikram College of Commerce, Patiala, Punjab 147004, India. Email: gaurav.dhiman@thapar.edu

Vijay Kumar, Computer Science and Engineering Department, National Institute of Technology, Hamirpur, India. Email: vijaykumarchahar@gmail.com
Genetic Algorithm (GA) and Differential Evolution (DE) are most popular of these methods. Some of evolutionarybased algorithms are: Evolution Strategy (ES), Biogeography-based Optimizer (BBO), and Genetic Programming (GP).

Swarm-based algorithms are kind of optimization algorithm that inspired from the foraging behaviors of insects, natural processes of plants, and social behaviors of animals. Particle Swarm Optimization (PSO), is inspired by the social movement of the birds. Ant Colony Optimization (ACO) is designed based on moving ants in order to select the shortest route. Some of other Swarm-based algorithms are: Emperor Penguin Optimizer (EPO), Grey Wolf Optimizer (GWO), Following Optimization Algorithm (FOA) [11], and Donkey Theorem Optimization (DTO) [12].

In this paper, Binary Orientation Search Algorithm (BOSA) is presented to optimize problems in various science.

Orientation Search Algorithm (OSA) has been explained in section 2. the Binary Orientation Search Algorithm (BOSA) is introduced In section 3. Then the results of BOSA are presented in section 4. Finally, in section 5, conclusions is expressed.

\section{ORIENTATION SEARCH ALGORITHM (OSA)} [13]

OSA simulates the rules of a game named orientation game. In this game players moves on the playground depending on the direction of the referee.

The initial position of the players shown in (1).

$$
X_{i}=\left(x_{i}^{1}, \ldots, x_{i}^{d}, \ldots, x_{i}^{n}\right)
$$

Here, $x_{i}^{d}$ is the position of dimension $d$ of player $i$, and $n$ is the number of variables.

In each iteration, the player who have best value of the fitness function is referee that is described in (2).

Referee $=$

$\{$ minimization problem: location of $\min (f i t)$

\{ maximization problem: location of $\max (f i t)$

Here, $f i t$ is the value of the fitness function.

Referee's hand direction is not necessarily the same as the referee's direction of motion. However, players must only consider the referee's hand. This direction is simulated in the form of Eqs. (3)-(4):

$$
\begin{aligned}
& P_{i}=0.8+0.2 \frac{t}{T} \\
& \text { Orintation }_{i}^{d} \\
& =\left\{\begin{array}{c}
\text { sign }\left(\text { Refere }^{d}-\text { Player }_{i}^{d}\right), \text { rand }<P_{i} \\
- \text { sign }\left(\text { Refere }^{d}-\text { Player }_{i}^{d}\right), \text { else }
\end{array}\right.
\end{aligned}
$$

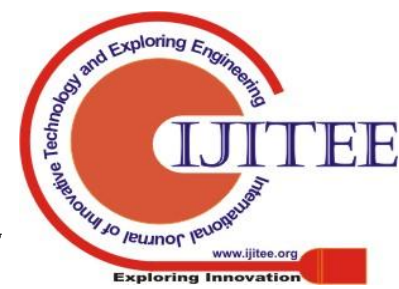


However, each player must move in the direction of the referee, some players may not be able to follow the referee. This issue is simulated in (5) and (6).

$$
\begin{aligned}
& \text { error }=0.2\left(1-\frac{t}{T}\right) \\
& X_{i}^{d} \\
& =\left\{\begin{array}{c}
X_{i}^{d}+\text { rand Orintation }{ }_{i}^{d} X_{h i}^{d}, \text { rand }>\text { error } \\
X_{l o}^{d}+\text { rand }\left(X_{h i}^{d}-X_{l o}^{d}\right), \quad \text { else }
\end{array}\right.
\end{aligned}
$$

At each iteration, the location of the players is updated by (1) to (6).

\section{BINARY ORIENTATION SEARCH ALGORITHM (BOSA)}

Although the Orientation Search Algorithm (OSA) is admissible for continuous problems, cannot be used it to binary problems. So, in this section, a binary model of this algorithm named Binary Orientation Search Algorithm (BOSA) is proposed.

In discrete space, each dimension of the particle position is represented by zero and one. The motion of an agent in any dimension means changing its value from zero to one or from one to zero. Therefore, the displacement of each dimension is calculated as a probability function which the player's position updates based this probability function. In BOSA, $d X^{j, d}$ is selected as the probability function limited in interval $[0-1]$. Equation (7) shows the probability function.

$$
S\left(d X^{j, d}(t)\right)=\left|\tanh \left(d X^{j, d}(t)\right)\right|
$$

The new position of each player based on the probability function is simulated in (8).

$$
\begin{gathered}
\text { If } \quad \operatorname{rand}<S\left(d X^{j, d}(t)\right) \quad \text { Then } \\
X^{j, d}(t+1)=\text { complement }\left(X^{j, d}(t)\right) \\
\text { Else } \quad X^{j, d}(t+1)=X^{j, d}(t)
\end{gathered}
$$

The different steps of Binary Orientation Search Algorithm (BOSA) are described below:

Step1. Defining system space and determining initial values.

Step2. The initial positioning of the players.

Step3. Evaluation of the players.

Step4. Determination of the direction by the referee for each player.

Step5. Updating the referee's position

Step6. Calculates the displacement probability function

Step7. Updating the player status.

Step8. Repeat steps 3 to 7 as long as the stop condition is met.

Step9. The end.

\section{RESULT AND DISCUSSION}

performance of BOSA is compared with other algorithm such as: Binary Grasshopper Optimization Algorithm (BGOA), Binary Dragonfly Algorithm (BDA), Binary Magnetic Optimization Algorithm (BMOA), Binary Bat Algorithm (BBA), Binary Gravitational Search Algorithm (BGSA), Binary Particle Swarm Optimization (BPSO), and Binary Genetic Algorithm (BGA) on twenty-three benchmark test.

\section{A. Benchmark test function}

This benchmark test function shown in table I to table III.

Table- I: Unimodal test functions

\begin{tabular}{|l|c|}
\hline$F_{1}(x)=\sum_{i=1}^{m} x_{i}^{2}$ & {$[-100,100]^{m}$} \\
\hline$F_{2}(x)=\sum_{i=1}^{m}\left|x_{i}\right|+\prod_{i=1}^{m}\left|x_{i}\right|$ & {$[-10,10]^{m}$} \\
\hline$F_{3}(x)=\sum_{i=1}^{m}\left(\sum_{j=1}^{i} x_{i}\right)^{2}$ & {$[-100,100]^{m}$} \\
\hline$F_{4}(x)=\max \left\{\left|x_{i}\right|, \quad 1 \leq i \leq m\right\}$ & {$[-100,100]^{m}$} \\
\hline$\left.F_{5}(x)=\sum_{i=1}^{m-1}\left[100\left(x_{i+1}-x_{i}^{2}\right)^{2}+\left(x_{i}-1\right)^{2}\right)\right]$ & {$[-30,30]^{m}$} \\
\hline$F_{6}(x)=\sum_{i=1}^{m}\left(\left[x_{i}+0.5\right]\right)^{2}$ & {$[-100,100]^{m}$} \\
\hline$F_{7}(x)=\sum_{i=1}^{m} i x_{i}^{4}+\operatorname{random}(0,1)$ & {$[-1.28,1.28]^{m}$} \\
\hline
\end{tabular}


Table- II: Multimodal test functions (type 1)

\begin{tabular}{|c|c|}
\hline$F_{8}(x)=\sum_{i=1}^{m}-x_{i} \sin \left(\sqrt{\left|x_{i}\right|}\right)$ & {$[-500,500]^{m}$} \\
\hline$F_{9}(x)=\sum_{i=1}^{m}\left[x_{i}^{2}-10 \cos \left(2 \pi x_{i}\right)+10\right]$ & {$[-5.12,5.12]^{m}$} \\
\hline$F_{10}(x)=-20 \exp \left(-0.2 \sqrt{\frac{1}{m} \sum_{i=1}^{m} x_{i}^{2}}\right)-\exp \left(\frac{1}{m} \sum_{i=1}^{m} \cos \left(2 \pi x_{i}\right)\right)+20+e$ & {$[-32,32]^{m}$} \\
\hline$F_{11}(x)=\frac{1}{4000} \sum_{i=1}^{m} x_{i}^{2}-\prod_{i=1}^{m} \cos \left(\frac{x_{i}}{\sqrt{i}}\right)+1$ & {$[-600,600]^{m}$} \\
\hline 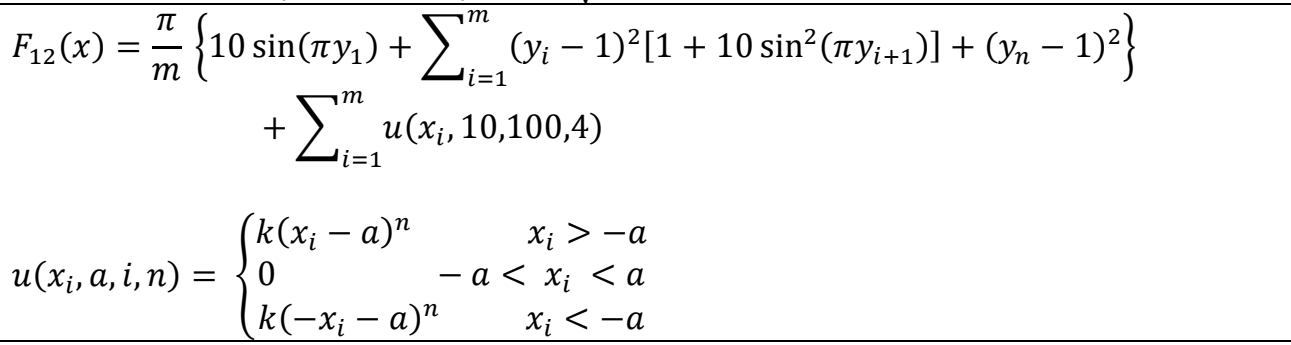 & {$[-50,50]^{m}$} \\
\hline $\begin{aligned} F_{13}(x)=0.1\{ & \sin ^{2}\left(3 \pi x_{1}\right) \\
& \left.+\sum_{i=1}^{m}\left(x_{i}-1\right)^{2}\left[1+\sin ^{2}\left(3 \pi x_{i}+1\right)\right]+\left(x_{n}-1\right)^{2}\left[1+\sin ^{2}\left(2 \pi x_{m}\right)\right]\right\} \\
& +\sum_{i=1}^{m} u\left(x_{i}, 5,100,4\right)\end{aligned}$ & {$[-50,50]^{m}$} \\
\hline
\end{tabular}

Table- III: Multimodal test functions (type 2)

\begin{tabular}{|l|c|}
\hline$F_{14}(x)=\left(\frac{1}{500}+\sum_{j=1}^{25} \frac{1}{j+\sum_{i=1}^{2}\left(x_{i}-a_{i j}\right)^{6}}\right)^{-1}$ & {$[-65.53,65.53]^{2}$} \\
\hline$F_{15}(x)=\sum_{i=1}^{11}\left[a_{i}-\frac{x_{1}\left(b_{i}^{2}+b_{i} x_{2}\right)}{b_{i}^{2}+b_{i} x_{3}+x_{4}}\right]^{2}$ & {$[-5,5]^{4}$} \\
\hline$F_{16}(x)=4 x_{1}^{2}-2.1 x_{1}^{4}+\frac{1}{3} x_{1}^{6}+x_{1} x_{2}-4 x_{2}^{2}+4 x_{2}^{4}$ & {$[-5,5]^{2}$} \\
\hline$F_{17}(x)=\left(x_{2}-\frac{5.1}{4 \pi^{2}} x_{1}^{2}+\frac{5}{\pi} x_{1}-6\right)^{2}+10\left(1-\frac{1}{8 \pi}\right) \cos x_{1}+10$ & {$[-5,10] \times[0,15]$} \\
\hline$F_{18}(x)=\left[1+\left(x_{1}+x_{2}+1\right)^{2}\left(19-14 x_{1}+3 x_{1}^{2}-14 x_{2}+6 x_{1} x_{2}+3 x_{2}^{2}\right)\right] \times\left[30+\left(2 x_{1}-3 x_{2}\right)^{2} \times(18\right.$ & {$[-5,5]^{2}$} \\
\hline$F_{19}(x)=-\sum_{i=1}^{4} c_{i} \exp \left(-\sum_{j=1}^{3} a_{i j}\left(x_{j}-P_{i j}\right)^{2}\right)$ & {$[0,1]^{3}$} \\
\hline$F_{20}(x)=-\sum_{i=1}^{4} c_{i} \exp \left(-\sum_{j=1}^{6} a_{i j}\left(x_{j}-P_{i j}\right)^{2}\right)$ & {$[0,1]^{6}$} \\
\hline$F_{21}(x)=-\sum_{i=1}^{5}\left[\left(X-a_{i}\right)\left(X-a_{i}\right)^{T}+6 c_{i}\right]^{-1}$ & {$[0,10]^{4}$} \\
\hline$F_{22}(x)=-\sum_{i=1}^{7}\left[\left(X-a_{i}\right)\left(X-a_{i}\right)^{T}+6 c_{i}\right]^{-1}$ & {$[0,10]^{4}$} \\
\hline$F_{23}(x)=-\sum_{i=1}^{10}\left[\left(X-a_{i}\right)\left(X-a_{i}\right)^{T}+6 c_{i}\right]^{-1}$ & {$[0,10]^{4}$} \\
\hline
\end{tabular}

\section{B. Simulation and discussion}

The functions $F_{1}-F_{7}$ are unimodal benchmark test function which are applied to evaluate the exploitation ability of the optimization algorithms. The optimization results for these functions are presented in table IV. BOSA has acceptable results compared to other algorithms in these types of test functions.

In multimodal functions of $F_{8}$ to $F_{13}$, by increasing the function dimensions, arriving at the minimum response of these functions is hardly possible. The optimization results for these functions are shown in table V. The results of this table show that BOSA Has good exploration power than other algorithms.

The functions F14 - F23 with a low number of dimensions have low local responses. The optimization results for these functions are shown in table VI.

These results show that the BOSA can be applied to solve optimization problems in various sciences. 
Table- IV: Unimodal test functions results

\begin{tabular}{|c|c|c|c|c|c|c|c|c|c|}
\hline & & BGA & BPSO & BGSA & BDA & BBA & BMOA & BGOA & BOSA \\
\hline \multirow{2}{*}{$\mathbf{F}_{1}$} & Ave & $1.95 \mathrm{E}-12$ & $4.98 \mathrm{E}-09$ & $1.16 \mathrm{E}-16$ & $2.81 \mathrm{E}-01$ & $7.86 \mathrm{E}-10$ & $4.61 \mathrm{E}-23$ & $5.71 \mathrm{E}-28$ & $6.74 \mathrm{E}-35$ \\
\hline & std & $2.01 \mathrm{E}-11$ & $1.40 \mathrm{E}-08$ & $6.10 \mathrm{E}-17$ & $1.11 \mathrm{E}-01$ & $8.11 \mathrm{E}-09$ & 7.37E-23 & $8.31 \mathrm{E}-29$ & $9.17 \mathrm{E}-36$ \\
\hline \multirow{2}{*}{$\mathbf{F}_{2}$} & Ave & $6.53 \mathrm{E}-18$ & $7.29 \mathrm{E}-04$ & $1.70 \mathrm{E}-01$ & $3.96 \mathrm{E}-01$ & 5.99E-20 & $1.20 \mathrm{E}-34$ & $6.20 \mathrm{E}-40$ & $7.78 \mathrm{E}-45$ \\
\hline & std & $5.10 \mathrm{E}-17$ & $1.84 \mathrm{E}-03$ & 9.29E-01 & $1.41 \mathrm{E}-01$ & $1.11 \mathrm{E}-17$ & $1.30 \mathrm{E}-34$ & $3.32 \mathrm{E}-40$ & $3.48 \mathrm{E}-45$ \\
\hline \multirow{2}{*}{$\mathbf{F}_{3}$} & Ave & $7.70 \mathrm{E}-10$ & $1.40 \mathrm{E}+01$ & $4.16 \mathrm{E}+02$ & $4.31 \mathrm{E}+01$ & $9.19 \mathrm{E}-05$ & $1.00 \mathrm{E}-14$ & $2.05 \mathrm{E}-19$ & $2.63 \mathrm{E}-25$ \\
\hline & std & 7.36E-09 & $7.13 \mathrm{E}+00$ & $1.56 \mathrm{E}+02$ & $8.97 \mathrm{E}+00$ & $6.16 \mathrm{E}-04$ & $4.10 \mathrm{E}-14$ & $9.17 \mathrm{E}-20$ & $9.83 \mathrm{E}-27$ \\
\hline \multirow{2}{*}{$\mathbf{F}_{4}$} & Ave & $9.17 \mathrm{E}+01$ & $6.00 \mathrm{E}-01$ & $1.12 \mathrm{E}+00$ & $8.80 \mathrm{E}-01$ & $8.73 \mathrm{E}-01$ & $2.02 \mathrm{E}-14$ & $4.32 \mathrm{E}-18$ & $4.65 E-26$ \\
\hline & std & $5.67 \mathrm{E}+01$ & $1.72 \mathrm{E}-01$ & $9.89 \mathrm{E}-01$ & $2.50 \mathrm{E}-01$ & $1.19 \mathrm{E}-01$ & $2.43 \mathrm{E}-14$ & $3.98 \mathrm{E}-19$ & $4.68 \mathrm{E}-29$ \\
\hline \multirow{2}{*}{$\mathbf{F}_{5}$} & Ave & $5.57 \mathrm{E}+02$ & $4.93 \mathrm{E}+01$ & $3.85 \mathrm{E}+01$ & $1.18 \mathrm{E}+02$ & $8.91 \mathrm{E}+02$ & $2.79 \mathrm{E}+01$ & $5.07 \mathrm{E}+00$ & $5.41 \mathrm{E}-01$ \\
\hline & std & $4.16 \mathrm{E}+01$ & $3.89 \mathrm{E}+01$ & $3.47 \mathrm{E}+01$ & $1.43 \mathrm{E}+02$ & $2.97 \mathrm{E}+02$ & $1.84 \mathrm{E}+00$ & $4.90 \mathrm{E}-01$ & $5.05 \mathrm{E}-02$ \\
\hline \multirow{2}{*}{$\mathbf{F}_{6}$} & Ave & $3.15 \mathrm{E}-01$ & $9.23 \mathrm{E}-09$ & $1.08 \mathrm{E}-16$ & $3.15 \mathrm{E}-01$ & $8.18 \mathrm{E}-17$ & $6.58 \mathrm{E}-01$ & 7.01E-19 & $8.03 \mathrm{E}-24$ \\
\hline & std & $9.98 \mathrm{E}-02$ & $1.78 \mathrm{E}-08$ & $4.00 \mathrm{E}-17$ & $9.98 \mathrm{E}-02$ & $1.70 \mathrm{E}-18$ & $3.38 \mathrm{E}-01$ & 4.39E-20 & $5.22 \mathrm{E}-26$ \\
\hline \multirow{2}{*}{$\mathbf{F}_{7}$} & Ave & $6.79 \mathrm{E}-04$ & $6.92 \mathrm{E}-02$ & $7.68 \mathrm{E}-01$ & $2.02 \mathrm{E}-02$ & $5.37 \mathrm{E}-01$ & $7.80 \mathrm{E}-04$ & $2.71 \mathrm{E}-05$ & 3.33E-08 \\
\hline & std & $3.29 \mathrm{E}-03$ & $2.87 \mathrm{E}-02$ & $2.77 \mathrm{E}+00$ & $7.43 \mathrm{E}-03$ & $1.89 \mathrm{E}-01$ & $3.85 \mathrm{E}-04$ & $9.26 \mathrm{E}-06$ & $1.18 \mathrm{E}-06$ \\
\hline
\end{tabular}

Table- V: Multimodal test functions result

\begin{tabular}{|c|c|c|c|c|c|c|c|c|c|}
\hline \multicolumn{2}{|c|}{} & BGA & BPSO & BGSA & BDA & BBA & BMOA & BGOA & BOSA \\
\hline \multirow{2}{*}{$\mathbf{F}_{\mathbf{8}}$} & Ave & $-5.11 \mathrm{E}+02$ & $-5.01 \mathrm{E}+02$ & $-2.75 \mathrm{E}+02$ & $-6.92 \mathrm{E}+02$ & $-4.69 \mathrm{E}+01$ & $-6.14 \mathrm{E}+02$ & $-8.76 \mathrm{E}+02$ & $-1.2 \mathrm{E}+04$ \\
\cline { 2 - 11 } & std & $4.37 \mathrm{E}+01$ & $4.28 \mathrm{E}+01$ & $5.72 \mathrm{E}+01$ & $9.19 \mathrm{EE}+01$ & $3.94 \mathrm{E}+01$ & $9.32 \mathrm{E}+01$ & $5.92 \mathrm{E}+01$ & $9.14 \mathrm{E}-12$ \\
\hline \multirow{2}{*}{$\mathbf{F}_{\mathbf{9}}$} & Ave & $1.23 \mathrm{E}-01$ & $1.20 \mathrm{E}-01$ & $3.35 \mathrm{E}+01$ & $1.01 \mathrm{E}+02$ & $4.85 \mathrm{E}-02$ & $4.34 \mathrm{E}-01$ & $6.90 \mathrm{E}-01$ & $8.76 \mathrm{E}-04$ \\
\cline { 2 - 11 } & std & $4.11 \mathrm{E}+01$ & $4.01 \mathrm{E}+01$ & $1.19 \mathrm{E}+01$ & $1.89 \mathrm{E}+01$ & $3.91 \mathrm{E}+01$ & $1.66 \mathrm{E}+00$ & $4.81 \mathrm{E}-01$ & $4.85 \mathrm{E}-02$ \\
\hline \multirow{2}{*}{$\mathbf{F}_{\mathbf{1 0}}$} & Ave & $5.31 \mathrm{E}-11$ & $5.20 \mathrm{E}-11$ & $8.25 \mathrm{E}-09$ & $1.15 \mathrm{E}+00$ & $2.83 \mathrm{E}-08$ & $1.63 \mathrm{E}-14$ & $8.03 \mathrm{E}-16$ & $8.04 \mathrm{E}-20$ \\
\cline { 2 - 11 } & std & $1.11 \mathrm{E}-10$ & $1.08 \mathrm{E}-10$ & $1.90 \mathrm{E}-09$ & $7.87 \mathrm{E}-01$ & $4.34 \mathrm{E}-07$ & $3.14 \mathrm{E}-15$ & $2.74 \mathrm{E}-14$ & $3.34 \mathrm{E}-18$ \\
\hline \multirow{2}{*}{$\mathbf{F}_{\mathbf{1 1}}$} & Ave & $3.31 \mathrm{E}-06$ & $3.24 \mathrm{E}-06$ & $8.19 \mathrm{E}+00$ & $5.74 \mathrm{E}-01$ & $2.49 \mathrm{E}-05$ & $2.29 \mathrm{E}-03$ & $4.20 \mathrm{E}-05$ & $4.23 \mathrm{E}-10$ \\
\cline { 2 - 11 } & std & $4.23 \mathrm{E}-05$ & $4.11 \mathrm{E}-05$ & $3.70 \mathrm{E}+00$ & $1.12 \mathrm{E}-01$ & $1.34 \mathrm{E}-04$ & $5.24 \mathrm{E}-03$ & $4.73 \mathrm{E}-04$ & $5.11 \mathrm{E}-07$ \\
\hline \multirow{2}{*}{$\mathbf{F}_{\mathbf{1 2}}$} & Ave & $9.16 \mathrm{E}-08$ & $8.93 \mathrm{E}-08$ & $2.65 \mathrm{E}-01$ & $1.27 \mathrm{E}+00$ & $1.34 \mathrm{E}-05$ & $3.93 \mathrm{E}-02$ & $5.09 \mathrm{E}-03$ & $6.33 \mathrm{E}-05$ \\
\cline { 2 - 11 } & std & $4.88 \mathrm{E}-07$ & $4.77 \mathrm{E}-07$ & $3.14 \mathrm{E}-01$ & $1.02 \mathrm{E}+00$ & $6.23 \mathrm{E}-04$ & $2.42 \mathrm{E}-02$ & $3.75 \mathrm{E}-03$ & $4.71 \mathrm{E}-04$ \\
\hline \multirow{2}{*}{$\mathbf{F}_{\mathbf{1 3}}$} & Ave & $6.39 \mathrm{E}-02$ & $6.26 \mathrm{E}-02$ & $5.73 \mathrm{E}-32$ & $6.60 \mathrm{E}-02$ & $9.94 \mathrm{E}-08$ & $4.75 \mathrm{E}-01$ & $1.25 \mathrm{E}-08$ & $0.00 \mathrm{E}+00$ \\
\cline { 2 - 10 } & std & $4.49 \mathrm{E}-02$ & $4.39 \mathrm{E}-02$ & $8.95 \mathrm{E}-32$ & $4.33 \mathrm{E}-02$ & $2.61 \mathrm{E}-07$ & $2.38 \mathrm{E}-01$ & $2.61 \mathrm{E}-07$ & $0.00 \mathrm{E}+00$ \\
\hline
\end{tabular}

Table- VI: Multimodal test functions with low dimension result

\begin{tabular}{|c|c|c|c|c|c|c|c|c|c|}
\hline & & BGA & BPSO & BGSA & BDA & BBA & BMOA & BGOA & BOSA \\
\hline \multirow{2}{*}{$\mathbf{F}_{14}$} & Ave & $4.39 \mathrm{E}+00$ & $2.77 \mathrm{E}+00$ & $3.61 \mathrm{E}+00$ & $9.98 \mathrm{E}+01$ & $1.26 \mathrm{E}+00$ & $3.71 \mathrm{E}+00$ & $1.08 \mathrm{E}+00$ & $9.98 \mathrm{E}-01$ \\
\hline & std & $4.41 \mathrm{E}-02$ & $2.32 \mathrm{E}+00$ & $2.96 \mathrm{E}+00$ & $9.14 \mathrm{E}-1$ & $6.86 \mathrm{E}-01$ & $3.86 \mathrm{E}+00$ & $4.11 \mathrm{E}-02$ & $7.64 \mathrm{E}-12$ \\
\hline \multirow{2}{*}{$\mathbf{F}_{15}$} & Ave & $7.36 \mathrm{E}-02$ & $9.09 \mathrm{E}-03$ & $6.84 \mathrm{E}-02$ & $7.15 \mathrm{E}-02$ & $1.01 \mathrm{E}-02$ & $3.66 \mathrm{E}-02$ & $8.21 \mathrm{E}-03$ & $3.3 \mathrm{E}-04$ \\
\hline & std & $2.39 \mathrm{E}-03$ & $2.38 \mathrm{E}-03$ & $7.37 \mathrm{E}-02$ & $1.26 \mathrm{E}-01$ & $3.75 \mathrm{E}-03$ & $7.60 \mathrm{E}-02$ & 4.09E-03 & $1.25 \mathrm{E}-05$ \\
\hline \multirow{2}{*}{$\mathbf{F}_{16}$} & Ave & $-1.02 \mathrm{E}+00$ & $-1.02 \mathrm{E}+00$ & $-1.02 \mathrm{E}+00$ & $-1.02 \mathrm{E}+00$ & $-1.02 \mathrm{E}+00$ & $-1.02 \mathrm{E}+00$ & $-1.02 \mathrm{E}+00$ & $-1.03 \mathrm{E}+00$ \\
\hline & std & 4.19E-07 & $0.00 \mathrm{E}+00$ & $0.00 \mathrm{E}+00$ & 4.74E-08 & $3.23 \mathrm{E}-05$ & 7.02E-09 & $9.80 \mathrm{E}-07$ & $5.12 \mathrm{E}-10$ \\
\hline \multirow{2}{*}{$\mathbf{F}_{17}$} & Ave & $3.98 \mathrm{E}-01$ & $3.98 \mathrm{E}-01$ & $3.98 \mathrm{E}-01$ & $3.98 \mathrm{E}-01$ & $3.98 \mathrm{E}-01$ & $3.98 \mathrm{E}-01$ & $3.98 \mathrm{E}-01$ & $3.98 \mathrm{E}-01$ \\
\hline & std & $3.71 \mathrm{E}-17$ & $9.03 \mathrm{E}-16$ & $1.13 \mathrm{E}-16$ & $1.15 \mathrm{E}-07$ & 7.61E-04 & $7.00 \mathrm{E}-07$ & 5.39E-05 & $4.56 \mathrm{E}-21$ \\
\hline \multirow{2}{*}{$\mathbf{F}_{18}$} & Ave & $3.00 \mathrm{E}+00$ & $3.00 \mathrm{E}+00$ & $3.00 \mathrm{E}+00$ & $3.00 \mathrm{E}+00$ & $3.00 \mathrm{E}+00$ & $3.00 \mathrm{E}+00$ & $3.00 \mathrm{E}+00$ & $3.00 \mathrm{E}+00$ \\
\hline & std & $6.33 \mathrm{E}-07$ & $6.59 \mathrm{E}-05$ & $3.24 \mathrm{E}-02$ & $1.48 \mathrm{E}+01$ & $2.25 \mathrm{E}-05$ & $7.16 \mathrm{E}-06$ & $1.15 \mathrm{E}-08$ & $1.15 \mathrm{E}-18$ \\
\hline \multirow{2}{*}{$\mathbf{F}_{19}$} & Ave & $-3.81 \mathrm{E}+00$ & $-3.80 \mathrm{E}+00$ & $-3.86 \mathrm{E}+00$ & $-3.77 \mathrm{E}+00$ & $-3.75 \mathrm{E}+00$ & $-3.84 \mathrm{E}+00$ & $-3.86 \mathrm{E}+00$ & $-3.86 \mathrm{E}+00$ \\
\hline & std & $4.37 \mathrm{E}-10$ & $3.37 \mathrm{E}-15$ & $4.15 \mathrm{E}-01$ & $3.53 \mathrm{E}-07$ & $2.55 \mathrm{E}-03$ & $1.57 \mathrm{E}-03$ & $6.50 \mathrm{E}-07$ & $5.61 \mathrm{E}-10$ \\
\hline \multirow{2}{*}{$\mathbf{F}_{20}$} & Ave & $-2.39 \mathrm{E}+00$ & $-3.32 \mathrm{E}+00$ & $-1.47 \mathrm{E}+00$ & $-3.23 \mathrm{E}+00$ & $-2.84 \mathrm{E}+00$ & $-3.27 \mathrm{E}+00$ & $-2.81 \mathrm{E}+00$ & $-3.31 \mathrm{E}+00$ \\
\hline & std & $4.37 \mathrm{E}-01$ & $2.66 \mathrm{E}-01$ & $5.32 \mathrm{E}-01$ & $5.37 \mathrm{E}-02$ & $3.71 \mathrm{E}-01$ & $7.27 \mathrm{E}-02$ & $7.11 \mathrm{E}-01$ & $4.29 \mathrm{E}-05$ \\
\hline \multirow{2}{*}{$\mathbf{F}_{21}$} & Ave & $-5.19 \mathrm{E}+00$ & $-7.54 \mathrm{E}+00$ & $-4.57 \mathrm{E}+00$ & $-7.38 \mathrm{E}+00$ & $-2.28 \mathrm{E}+00$ & $-9.65 \mathrm{E}+00$ & $-8.07 \mathrm{E}+00$ & $-10.15 \mathrm{E}+00$ \\
\hline & std & $2.34 \mathrm{E}+00$ & $2.77 \mathrm{E}+00$ & $1.30 \mathrm{E}+00$ & $2.91 \mathrm{E}+00$ & $1.80 \mathrm{E}+00$ & $1.54 \mathrm{E}+00$ & $2.29 \mathrm{E}+00$ & $1.25 \mathrm{E}-02$ \\
\hline \multirow{2}{*}{$\mathbf{F}_{22}$} & Ave & $-2.97 \mathrm{E}+00$ & $-8.55 \mathrm{E}+00$ & $-6.58 \mathrm{E}+00$ & $-8.50 \mathrm{E}+00$ & $-3.99 \mathrm{E}+00$ & $-1.04 \mathrm{E}+00$ & $-10.01 \mathrm{E}+00$ & $-10.40 \mathrm{E}+00$ \\
\hline & std & $1.37 \mathrm{E}-02$ & $3.08 \mathrm{E}+00$ & $2.64 \mathrm{E}+00$ & $3.02 \mathrm{E}+00$ & $1.99 \mathrm{E}+00$ & $2.73 \mathrm{E}-04$ & $3.97 \mathrm{E}-02$ & $3.65 \mathrm{E}-07$ \\
\hline \multirow{2}{*}{$\mathbf{F}_{23}$} & Ave & $-3.10 \mathrm{E}+00$ & $-9.19 \mathrm{E}+00$ & $-9.37 \mathrm{E}+00$ & $-8.41 E+00$ & $-4.49 \mathrm{E}+00$ & $-1.05 \mathrm{E}+01$ & $-3.41 \mathrm{E}+00$ & $-10.53 E+00$ \\
\hline & std & $2.37 \mathrm{E}+00$ & $2.52 \mathrm{E}+00$ & $2.75 \mathrm{E}+00$ & $3.13 \mathrm{E}+00$ & $1.96 \mathrm{E}+00$ & $1.81 \mathrm{E}-04$ & $1.11 \mathrm{E}-02$ & $5.26 \mathrm{E}-06$ \\
\hline
\end{tabular}




\section{CONCLUSION}

These days Random-based algorithms are applied for optimization in different science widely. Most of these algorithms are inspired by beings' behavior or physical processes. In this paper, a binary model for Orientation Search Algorithm named Binary Orientation Search Algorithm (BOSA), based on the governing rules of a game, is introduced. The players in this game try to find the optimal response with the influence from each other and the referee.

The performance of BOSA has been evaluated by twentythree benchmark test functions. The results indicates that BOSA supply very competitive results as compared with other optimization algorithm such as BGA, BPSO, BGSA, BBA, BMOA, BDA, and BGOA. The results on tree types of test functions shows that BOSA has acceptable results compared to other algorithms.

\section{REFERENCES}

1. M. DEHGHANI, Z. MONTAZERI, and O. P. MALIK, "DGO: Dice Game Optimizer," Gazi University Journal of Science, vol. 32, pp. 871$882,2019$.

2. M. Dehghani, Z. Montazeri, and O. Malik, "Energy commitment: a planning of energy carrier based on energy consumption," Электротехника и электромеханика, 2019.

3. Z. Montazeri and T. Niknam, "OPTIMAL UTILIZATION OF ELECTRICAL ENERGY FROM POWER PLANTS BASED ON FINAL ENERGY CONSUMPTION USING GRAVITATIONAL SEARCH ALGORITHM," 2018, p. 4, 2018-08-17 2018.

4. S. Dehbozorgi, A. Ehsanifar, Z. Montazeri, M. Dehghani, and A. Seifi, "Line loss reduction and voltage profile improvement in radial distribution networks using battery energy storage system," in 2017 IEEE 4th International Conference on Knowledge-Based Engineering and Innovation (KBEI), 2017, pp. 0215-0219.

5. M. Dehghani, M. Mardaneh, Z. Montazeri, A. Ehsanifar, M. Ebadi, and O. Grechko, "Spring search algorithm for simultaneous placement of distributed generation and capacitors," Электротехника u электромеханика, 2018.

6. M. Dehghani, Z. Montazeri, A. Ehsanifar, A. Seifi, M. Ebadi, and O. Grechko, "Planning of energy carriers based on final energy consumption using dynamic programming and particle swarm optimization," Электротехника и электромеханика, 2018.

7. Z. Montazeri and T. Niknam, "Energy carriers management based on energy consumption," in Knowledge-Based Engineering and Innovation (KBEI), 2017 IEEE 4th International Conference on, 2017, pp. 0539-0543.

8. S. Kirkpatrick, C. D. Gelatt, and M. P. Vecchi, "Optimization by simulated annealing," science, vol. 220, pp. 671-680, 1983.

9. M. Dehghani, Z. Montazeri, A. Dehghani, N. Nouri, and A. Seifi, "BSSA: Binary spring search algorithm," in Knowledge-Based Engineering and Innovation (KBEI), 2017 IEEE 4th International Conference on, 2017, pp. 0220-0224.

10. M. Dehghani, Z. Montazeri, A. Dehghani, and A. Seifi, "Spring search algorithm: A new meta-heuristic optimization algorithm inspired by Hooke's law," in Knowledge-Based Engineering and Innovation (KBEI), 2017 IEEE 4th International Conference on, 2017, pp. 02100214.

11. M. Dehghani, M. Mardaneh, and O. Malik, "FOA:"Following'Optimization Algorithm for solving Power engineering optimization problems," Journal of Operation and Automation in Power Engineering, 2019.

12. M. Dehghani, M. Mardaneh, O. P. Malik, and S. M. NouraeiPour, "DTO: Donkey Theorem Optimization," in 2019 27th Iranian Conference on Electrical Engineering (ICEE), 2019, pp. 1855-1859.

13. M. Dehghani, Z. Montazeri, O. P. Malik, A. Ehsanifar, and A. Dehghani, "OSA: Orientation Search Algorithm," International Journal of Industrial Electronics, Control and Optimization, pp. -, 2018.

\section{AUTHORS PROFILE}

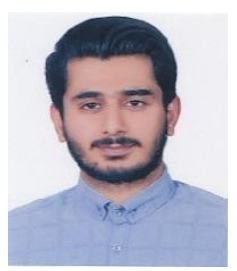

energy carriers.

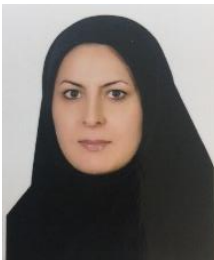

Zeinab Montazeri received a B.S. degree in Electrical Engineering from Electrical Engineering Islamic Azad University of Marvdasht. Marvdasht, I.R. Iran, in 2016 and M.Sc. degree in Electrical Engineering Islamic Azad University of Marvdasht, Marvdasht, I.R. Iran, in 2018. She has been PhD. Student at Shiraz University of Technology, Shiraz, Iran, since 2018. Her curren research interests include optimization algorithms; micro girds power systems, energy, and energy carriers.

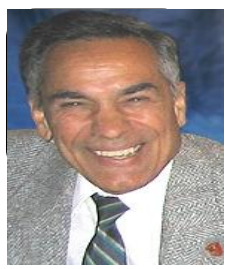

Om Parkash Malik received the M.E. degree in Electric Machine Design from Roorkee University, India, in 1962, the Ph.D. degree from the Imperial College, University of London, London, U.K., in 1965. He is currently Professor Emeritus with the Department of Electrical and Computer Engineering, University of Calgary, Calgary, AB, Canada. He has also done extensive research in the development and application of adaptive and artificial intelligence-based techniques for realtime control and protection of machines and power systems.

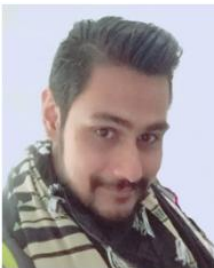

Gaurav Dhiman received his Ph.D. in Computer Engineering from Thapar Institute of Engineering \& Technology, Patiala. He has completed his Master Degree of Computer Applications from Thapar Institute of Engineering \& Technology, Patiala. His research interest includes Single, and Multi objectives optimization, Soft computing, Power systems, and Change detection using remotely sensed highresolution satellite data.

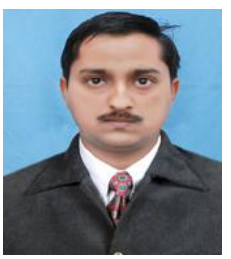

Vijay Kumar received the B.Tech. from M.M. Engineering College, Mullana. He received Ph.D. and M.Tech. from National Institute of Technology, Kurukshetra and Guru Jambheshwer University of Science and Technology, Hisar, respectively. His main research focuses on Soft Computing, Image Processing, and Data Clustering and Multi objective

optimization. 